\title{
SPREAD AND LOCAL PROPERTIES
}

\author{
BASIL COUTANT
}

(Communicated by Dennis Burke)

\begin{abstract}
A technique for combining the spread of a space and several local properties is exploited to obtain decompositions of a space and cardinal function bounds on the size and weight. It is shown that a locally countable set is the union of $\omega_{1}$ discrete sets and that under $M A\left(\omega_{1}\right)$ the weight of a manifold is equal to its spread.
\end{abstract}

\section{INTRODUCTION}

The main technique used in this paper is taken from a lemma the author used to prove that $s\left[C_{p}(X)^{\omega}\right] \leq s\left[C_{p}(X)\right]^{+}\left(C_{p}(X)=\right.$ space of continuous realvalued functions with the topology of pointwise convergence) [3]. It is a variant of a well-known (and useful) fact about CCC spaces: if $\mathscr{U}$ is an open collection, then there is a countable subcollection $\mathscr{V}$ such that the union of $\mathscr{V}$ is dense in the union of $\mathscr{U}$ [4], [6]. This fact is easily proven by considering a maximal cellular open refinement. The method used here is to repeatedly take maximal cellular subcollections and then to use the knowledge of a local property to show when this process stops. This avoids using closure to get a cover of the original open collection. The applications often yield bounds in terms of a successor cardinal, as in the inequality for $C_{p}(X)$ above. Not surprisingly, the sharpness of the bounds depends on extra set-theoretic assumptions.

We start by considering subsets of a space which are locally $<\lambda$ for some infinite cardinal $\lambda$ (each point has a relatively open neighborhood of cardinality $<\lambda)$.

1.1. Theorem. If $A \subseteq X$ is locally $<\lambda$, then $A$ is the union of $\lambda$ discrete subsets.

Proof. The proof may be achieved with relatively open sets, so without loss of generality assume that $A=X$. Let $\mathscr{V}=\left\{V_{x}: x \in X\right\}$ be a collection of open

Received by the editors December 6, 1989 and, in revised form, March 2, 1990.

1980 Mathematics Subject Classification (1985 Revision). Primary 54A25; Secondary 54A35, 03E50, 57N60.

Key words and phrases. Cardinality of a space, locally countable, locally second countable, nonmetrizable manifolds, spread, $S / L$-spaces, weight of a space. 
neighborhoods such that, for each $x \in X$,

(1) $x \in V_{x}$ and

(2) $\left|V_{x}\right|<\lambda$.

For $\alpha \in \lambda$ inductively define sets $A_{\alpha}$ and $S_{\alpha}$ such that

(a) $S_{\alpha} \subseteq A_{\alpha} \subseteq X \backslash \bigcup_{\beta \in \alpha} A_{\beta}$;

(b) $S_{\alpha}$ is discrete;

(c) if $X \backslash\left(\bigcup_{\beta \in \alpha} A_{\beta}\right)$ is nonempty, then $\left\{V_{x} \backslash\left(\bigcup_{\beta \in \alpha} A_{\beta}\right): x \in S_{\alpha}\right\}$ is a maximal pairwise disjoint collection in $X \backslash\left(\bigcup_{\beta \in \alpha} A_{\beta}\right)$, and $A_{\alpha}=$ $\bigcup\left\{V_{x}: x \in S_{\alpha}\right\} \backslash\left(\bigcup_{\beta \in \alpha} A_{\beta}\right)$.

If $X \backslash\left(\bigcup_{\beta \in \alpha} A_{\beta}\right)$ is empty, then $S_{\alpha}=A_{\alpha}=\varnothing$.

Claim. $\bigcup\left\{A_{\alpha}: \alpha \in \lambda\right\}=X$.

Assume the contrary, and let $x \in X \backslash\left(\bigcup_{\beta \in \lambda} A_{\beta}\right)$. Then, by the maximality in (c), we have that $V_{x}$ meets $A_{\alpha}$ for each $\alpha \in \lambda$. However, condition (a) causes the $A_{\alpha}$ 's to be pairwise disjoint. Hence the cardinality of $V_{x}$ must be at least $\lambda$, which is a contradiction.

Now we can define the discrete subspaces. Fix $\alpha \in \lambda . A_{\alpha}$ is covered by the pairwise disjoint, relatively open collection $\left\{V_{x} \backslash\left(\bigcup_{\beta \in \alpha} A_{\beta}\right): x \in S_{\alpha}\right\}$. Furthermore, each $V_{x} \backslash\left(\bigcup_{\beta \in \alpha} A_{\beta}\right)$ can be written as $\left\{z_{x, \beta}: \beta \in \lambda\right\}$ (repeat an element). So, for each $\beta \in \lambda$, let $D_{\alpha, \beta}=\left\{z_{x, \beta}: x \in S_{\alpha}\right\}$. Then for each $y \in S_{\alpha}, D_{\alpha, \beta} \cap V_{y}=\left\{z_{y, \beta}\right\}$. Hence $D_{\alpha, \beta}$ is discrete. So, by the claim, $X=\bigcup\left\{D_{\alpha, \beta}: \alpha, \beta \in \lambda\right\}$ is the union of $\lambda$ discrete sets.

Theorem 1.1 also has the following set-theoretic version:

1.2. Theorem. Let $\mathscr{V} \subseteq[E]^{<\lambda}$ be a cover of a set $E$. Then there is a partition $\left\{A_{\alpha}: \alpha \in \lambda\right\}$ of $E$ and a sequence of subcollections of $\mathscr{V},\left\{\mathscr{V}_{\alpha}: \alpha \in \lambda\right\}$, such that $\left\{V \cap A_{\alpha}: V \in \mathscr{V}_{\alpha}\right\}$ is a partition of $A_{\alpha}$.

1.3. Corollary. If $X$ is locally $<\lambda$, then $|X| \leq \lambda \cdot s(X)$. (Hence locally countable $S$-spaces have cardinality at most $\omega_{1}$.)

The study of $S$-spaces often begins by reducing the problem to a subset of $2^{\omega_{1}}$. By standard techniques, if $X$ is an $S$-space, then $X$ contains a locally countable $S$-subspace. Consequently under $C H$ it contains a locally countable $S$-subspace of weight at most $\omega_{1}$.

1.4. Corollary. If $X$ is a locally countable Tychonoff space with countable spread and $\chi(X) \leq \omega_{1}$, then $w(X) \leq \omega_{1}$, and hence $X$ can be embedded in $2^{\omega_{1}}$. (Consequently all the locally compact [or $1^{\text {st }}$ countable], locally countable $S$-spaces can be embedded in $2^{\omega_{1}}$.)

Proof. $w(X) \leq|X| \cdot \chi(X)$. 
As a comparison to Theorem 1.1, consider the following theorem of Balogh: 1.5. Theorem (Balogh [2]). [MA+ $7 C H]$ In a compact space $X$ of countable tightness, every locally countable subset of cardinality $<2^{\omega}$ is the countable union of discrete sets.

\section{RESULTS USING HEREDITARY LINDELÖF DEGREE}

In this section, the cardinality condition in Theorem 1.1 is replaced with the hereditary Lindelöf degree with similar results. In fact, under $\mathrm{CH}$ we find that local countability can be weakened to locally hereditarily Lindelöf in Theorem 1.1 .

2.1. Theorem. If a $T_{2}$ space $X$ is locally hereditarily $\lambda$-Lindelöf, then $X$ has an open cover $\mathscr{V}=\bigcup\left\{\mathscr{V}_{\alpha}: \alpha \in \lambda^{+}\right\}$of hereditarily $\lambda$-Lindelöf sets such that $\forall \alpha \in \lambda^{+}, \mathscr{V}_{\alpha}$ witnesses a discrete set $S_{\alpha}$. Also, $X$ can be written as the union of $2^{\lambda}$ discrete sets.

Proof. Fix an open cover $\mathscr{W}=\left\{V_{x}: x \in X\right\}$ such that for each $x \in X, x \in V_{x}$ and $h L\left(V_{x}\right) \leq \lambda$. For $\alpha \in \lambda^{+}$, inductively define the sets $A_{\alpha}$ and $S_{\alpha}$ such that

(a) $S_{\alpha} \subseteq A_{\alpha} \subseteq X \backslash \bigcup_{\beta \in \alpha} A_{\beta}$;

(b) $S_{\alpha}$ is discrete;

(c) if $X \backslash\left(\bigcup_{\beta \in \alpha} A_{\beta}\right)$ is nonempty, then $\left\{V_{x} \backslash\left(\bigcup_{\beta \in \alpha} A_{\beta}\right): x \in S_{\alpha}\right\}$ is a maximal pairwise disjoint collection in $X \backslash\left(\bigcup_{\beta \in \alpha} A_{\beta}\right)$ and $A_{\alpha}=$ $\bigcup\left\{V_{x}: x \in S_{\alpha}\right\} \backslash\left(\bigcup_{\beta \in \alpha} A_{\beta}\right)$.

If $X \backslash\left(\bigcup_{\beta \in \alpha} A_{\beta}\right)$ is empty, then $S_{\alpha}=A_{\alpha}=\varnothing$.

Claim. $X=\bigcup\left\{A_{\alpha}: \alpha \in \lambda^{+}\right\}$, and hence $X$ is covered by $\bigcup\left\{\mathscr{V}_{\alpha}: \alpha \in \lambda^{+}\right\}$, where $\mathscr{V}_{\alpha}=\left\{V_{x}: x \in S_{\alpha}\right\}$.

Assume the contrary, and let $x \in X \backslash \bigcup\left\{A_{\alpha}: \alpha \in \lambda^{+}\right\}$. Consider the following collection:

$$
\left\{\left(\bigcup_{\beta \in \alpha} A_{\beta}\right) \cap V_{x}: \alpha \in \lambda^{+}\right\} .
$$

As in Theorem 1.1, $V_{x} \cap A_{\alpha} \neq \varnothing$ for each $\alpha \in \lambda^{+}$by the maximality in (c). Hence the above collection $(*)$ is strictly increasing. Also note that by the definition of the $A_{\beta}$ 's for each $\alpha \in \lambda^{+}$,

$$
\bigcup_{\beta \in \alpha} A_{\beta}=\bigcup\left\{V_{y}: y \in \bigcup_{\beta \in \alpha} S_{\beta}\right\},
$$

and hence is open. Therefore $(*)$ is a strictly increasing sequence of open sets in $V_{x}$ contradicting the assumption that $h L\left(V_{x}\right) \leq \lambda$.

Recall that for any $T_{2}$ space $Y,|Y| \leq 2^{h L(Y)}$ (see Hodel [6]). So for each $\alpha \in \lambda^{+}$and $x \in S_{\alpha}$ write each $V_{x} \backslash\left(\bigcup_{\beta \in \alpha} A_{\beta}\right)$ as $\left\{z_{x, \gamma}: \gamma \in 2^{\lambda}\right\}$ (repeat an 
element if necessary). Then, as in Theorem 1.1, the set $D_{\alpha, \gamma}=\left\{z_{x, \gamma}: x \in S_{\alpha}\right\}$ is discrete and $X=\bigcup\left\{D_{\alpha, \gamma}: \alpha \in \lambda^{+} \wedge \gamma \in 2^{\lambda}\right\}$.

2.2. Theorem. If $X$ is a $T_{2}$ space with an open cover $\mathscr{V}$ of sets with hereditary Lindelöf degree at most $\lambda$, then $h L(X) \leq \lambda^{+} \cdot s(X)$ and $|X| \leq 2^{\lambda} \cdot s(X)$.

2.3. Theorem. Let $\lambda<\kappa$ be infinite cardinals. If each point $x$ in a $T_{2}$ space $X$ has a neighborhood $V_{x}$ such that $h L\left(V_{x}\right) \leq \lambda$ and $w\left(V_{x}\right) \leq \kappa$, then $w(X) \leq$ $\kappa \cdot s(X)$.

Proof. Let $\mathscr{V}=\left\{V_{\alpha}: \alpha \in \lambda^{+} \cdot s(X)\right\}$ be an open cover of sets of weight at most $\kappa$. Fix a base $\mathscr{B}_{\alpha}$ of cardinality $\leq \kappa$ for each $V_{\alpha} \in \mathscr{V}$. Then, since each $V_{\alpha}$ is open and $X=\bigcup \mathscr{V}, \mathscr{B}=\bigcup\left\{\mathscr{B} \alpha, \alpha \in \lambda^{+} \cdot s(X)\right\}$ is a base for the entire space of cardinality, at most $\lambda^{+} \cdot \kappa \cdot s(X)=\kappa \cdot s(X)$.

2.4. Corollary. If $X$ is locally compact, then $w(X) \leq \Delta(X)^{+} \cdot s(X)$ and $|X| \leq$ $2^{\Delta(X)} \cdot s(X)$. In particular, if $X$ has a $G_{\delta}$-diagonal, then $w(X) \leq \omega_{1} \cdot s(X)$ and $|X| \leq 2^{\omega} \cdot s(X)$.

Proof. For a compact neighborhood $V_{x}$, we have $h L\left(V_{x}\right) \leq w\left(V_{x}\right) \leq \Delta(X)$. So let $\lambda=\Delta(X)$ and $\kappa=\Delta(X)^{+}$in Theorems 2.2 and 2.3.

2.5. Corollary. For any locally metrizable space, $w(X) \leq s(X)^{+}$and $|X| \leq$ $2^{s(X)}$.

Proof. For a metrizable $V, h L(V)=w(V) \leq s(X)$. So let $\lambda=s(X)$ and $\kappa=s(X)^{+}$in Theorems 2.2 and 2.3.

2.6. Corollary. If $X$ is locally second countable such that $s(X) \neq w(X)$, then $s(X)=\omega$ and $w(X)=\omega_{1}$.

It is known that $h d(X)=s(X) \leq L(X)=w(X)$ for any manifold $X$. In addition, M. E. Rudin has constructed a manifold under $\diamond$ with countable spread and uncountable weight. (See [8].) Hence Corollaries 2.5 and 2.6 are the best possible $Z F C$ statements. Under $M A\left(\omega_{1}\right)$, however, for any manifold we have equality for each of the four cardinals.

2.7. Theorem $\left[M A\left(\omega_{1}\right)\right]$. If a space $X$ is both locally compact and locally metrizable, then $s(X)=w(X)$ (i.e., it is consistent that $s(X)=w(X)$ for all manifolds).

Proof. Use the fact that under $M A\left(\omega_{1}\right)$ there are no locally compact $S$-spaces. (See Balogh's paper [2].)

2.8. Theorem. If there is a space $X$ of countable spread such that $X$ is either

(a) locally compact with a $G_{\delta}$-diagonal and $w(X)=2^{\omega}$; or

(b) locally metrizable (second countable) with $w(X)=2^{\omega}$; or

(c) locally countable with $|X|=2^{\omega}$,

then the Continuum Hypothesis holds. 
2.9. Example (Kunen [7]). ( $C H)$ The Kunen line is a locally countable, locally compact, locally metrizable space $X$ with countable spread, a $G_{\delta}$-diagonal, and $w(X)=|X|=2^{\omega}$.

As an alternate view of the above, consider the fact that it is consistent with $M A+7 C H$ that there is a first countable $S$-space but there are no first countable $L$-spaces (see [1] and [9]). In both cases we have $s(X)=\omega$; hence, the following applies equally to both situations.

2.10. Theorem. If $s(X)=\omega$ and $w(X) \geq \omega_{2}\left(\right.$ or $w(X) \geq 2^{\omega}$ under $\left.7 C H\right)$, then $X$ contains a point with no second countable neighborhood.

We conclude this section with several results of Balogh which use hereditary normality and Jones' lemma to provide a bound on the spread. For these we will need extra set-theoretic assumptions. Let $(\mathscr{E})$ be the following statement about the exponential function implied by both $M A$ and $C H$ :

$$
\text { for each cardinal } \kappa, \kappa<2^{\omega} \Rightarrow 2^{\kappa}<2^{2^{\omega}} \text {. }
$$

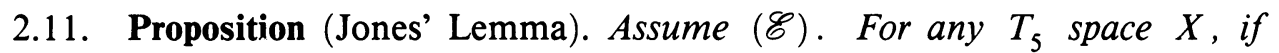
$d(X)<2^{\omega}$, then $X$ has no discrete sets of size $2^{\omega}$.

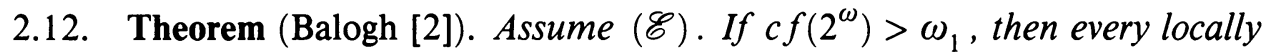
hereditarily Lindelöf, $T_{5}$ of density $<2^{\omega}$ has Lindelöf degree $<2^{\omega}$.

Proof. For each $x \in X$, fix a hereditarily Lindelöf neighborhood $V_{x}$. By Theorem 2.1, there is a subcollection, $\mathscr{W}$, of these neighborhoods such that

(1) $\mathscr{W}=\bigcup\left\{\mathscr{W}_{\alpha}: \alpha \in \omega_{1}\right\}$ is a cover of $X$ and

(2) each $\mathscr{W}_{\alpha}$ witnesses a discrete set.

By Jones' Lemma, $\left|\mathscr{W}_{\alpha}\right|<2^{\omega}$ for each $\alpha$, and by the cofinality restriction, $|\mathscr{W}|<2^{\omega}$.

2.13. Theorem (Balogh [2]). Assume ( $\mathscr{E})$. If $c f\left(2^{\omega}\right)>\omega_{1}$, then every point in a locally hereditarily Lindelöf, locally separable, $T_{5}$ space is contained in a clopen set of Lindelöf degree $<2^{\omega}$.

Proof. For each $x \in X$ construct, using Theorem 2.12, an increasing sequence of open sets $\left\{W_{\alpha}: \alpha \in \omega_{1}\right\}$ containing $x$ such that for each $\alpha \in \omega_{1}$

(a) $\overline{W_{\alpha}} \subseteq W_{\alpha+1}$ and

(b) $d\left(W_{\alpha}\right)<2^{\omega}$ (local separability and Theorem 2.12).

Then the union will be clopen by the local separability and will have Lindelöf degree $<2^{\omega}$ by Theorem 2.12 .

2.14. Remarks. (i) Balogh assumes the space is locally hereditarily separable, but needs to consider only the density of open sets. See his paper [2] and Fremlin's [5] for more discussion on the above theorem and the consequences of combining $M A$ or $P F A$ with local properties. 
(ii). In Theorems 1.1 and 2.1 , we can fix the open cover $\mathscr{V}$ that we are working with first, and then replace the spread of $X$ with a bound on the size of a disjoint subcollection of $\{V \cap A: V \in \mathscr{V}\}$, where $A \subseteq \cup \mathscr{V}$. This was necessary to prove that $s\left[C_{p}(X)^{\omega}\right] \leq s\left[C_{p}(X)\right]^{+}[3]$.

\section{ACKNOWLEDGMENT}

The author wishes to thank the referee for calling attention to Theorem 1.2.

\section{REFERENCES}

1. U. Abraham and S. Todorčević, Martin's axiom and first-countable S- and L-spaces, Handbook of Set Theoretic Topology, North-Holland, Amsterdam, 1984, pp. 327-346.

2. Z. Balogh, Locally nice spaces under Martin's axiom, Comment Math. Univ. Carolin. 24 (1983), 63-87.

3. B. Coutant, Some cardinal function relationships between $C_{p}(X)$ and finite powers of a space $X$, Topology Appl. (to appear).

4. R. Engelking, General topology, Polish Scientific Publishers, Warsaw, 1977.

5. D. Fremlin, Perfect pre-images of $\omega_{1}$ and the PFA, Topology Appl. 29 (1988), 151-166.

6. R. Hodel, Cardinal functions I, Handbook of Set Theoretic Topology, North-Holland, Amsterdam, 1984, pp. 63-109.

7. I. Juhász, K. Kunen, and M. E. Rudin, Two more hereditarily separable non-Lindelöf spaces, Canad. J. Math. 28 (1976), 998-1005.

8. P. Nyikos, The theory of nonmetrizable manifolds, Handbook of Set Theoretic Topology, North-Holland, Amsterdam, 1984, pp. 633-684.

9. J. Roitman, Basic $S$ and L, Handbook of Set Theoretic Topology, North-Holland, Amsterdam, 1984, pp. 295-326.

Department of Mathematics, Ohio University, Athens, Ohio 45701

Current address: Department of Mathematical Sciences, University of Alaska-Fairbanks, Fairbanks, Alaska 99775 